\title{
Design of Low Power Spy Type Wireless GPS Tracking Terminal System
}

\author{
Zhenming Bao ${ }^{1}$, Qi Wang ${ }^{2}$, Qiancheng Zhao ${ }^{1,3}$ and Jieyang Ruan ${ }^{3}$ \\ ${ }^{1}$ Jiangsu university science and technology, Zhenjiang, China \\ ${ }^{2}$ Jiangsu university science and technology, Zhangjiagang,China \\ ${ }^{3}$ Shanghai NAEN Automotive Technology Co., Ltd, China
}

\begin{abstract}
Low power spy type wireless GPS tracking terminal is a positioning device which can receive space GPS signal by the host tracker, through GPS signal analysis, their own geodetic coordinate can be calculated, then the device will report its positioning information to monitoring center by GPRS wireless network. It uses the advanced global positioning (GPS) technique to obtain the position of the vehicle information and velocity information; the mature mobile communication technology will ensure data to be sent to the client timely. This design chooses ultra-low power MCU, and then the design and optimization for power supply scheme makes the whole system meet the requirements of low power consumption of super-long standby. At the same times, the test for the prototype shows that it meets the design requirements.
\end{abstract}

Keywords-GPS; GPRS; mobile communications; low power tracker

\section{INTRODUCTION}

Intelligent Vehicle Tracking System (IVTS) is mainly used for vehicle tracking and navigation. It uses the world's leading global satellite positioning technology, global mobile communication technology, geographic information processing technology and computer network communications and data processing technology ${ }^{[1,2]}$. With the continuous promotion of navigation and positioning technology, it now has become a standard configuration of a car. But the positioning system in most vehicles uses the inner power supply and its position is fixed. It can easily be found and removed according to the car power alignments.

With the development of banks' car mortgage and P2P car loan business, to prevent lenders from cheating loans and avoiding debt liability, car loan companies need to install a wired GPS positioning system for their cars and the loan contract is the constraint of installing GPS. While installing the cable GPS positioning equipment, and then concealed mounting one or two wireless GPS tracking device in other location of the vehicle. Once there is equipment removed, the device will send a warning message to the monitoring terminal. If the behavior belongs to cheating loans or avoiding debt liability after verification, the company can remotely start wireless GPS device and start continuous positioning, and finding the vehicle in accordance with the position of the wireless device. But the main drawbacks of the existing wireless GPS devices on the market are the high price, oversized volume, not hidden, short standby time and so on, therefore a vehicle positioning terminal product with a long standby time, small volume, and easy installation is needed. A low-cost and ultra-low-power spy type wireless GPS tracking terminal system is designed in this thesis.

\section{System PROGRAM DESIGN}

The system uses STM8L151F3 from STMicroelectronics as the core control unit, M26 (GSM) module and L70-R (GPS) module from Shanghai QUECTEL Company are used as mobile communications and positioning unit respectively. L70-R supports the NMEA 0183 standard command and control, the main control unit controls GPS module by sending standard NMEA0183 commands to obtain position, velocity and time information. The main controller will forward information to the M26, the M26 module is embedded TCP / IP communication protocol, main control unit can achieved a TCP connection by AT commands and send information to the remote server. After receiving information, the server can quickly locate the vehicle combined with the location information of cellular base stations. Comparing that the traditional GPS positioning needs 2 or 3 minutes for the first time, the fastest time to first fix of the location base station (LBS) needs only a few seconds, at the same time, the assisted positioning of base station also completely solve the defect that common GPS device can't locate in the room ${ }^{[3]}$.

In addition to the above-described function modules, the system also adds functions about low-voltage detection and alarming and anti-demolition detection. Low voltage signal is collected through the microcontroller $\mathrm{AD}$ port, when the voltage drops below a preset threshold, the main control unit will send a low pressure warning to the remote server. Anti-demolition function is to use a photoresistor to sense the change of external light, the photoresistor is encapsulated by shell. At the bottom of the shell left a light transmission hole, the hole is blocked when the device is mounted. When the device is removed, light transmission hole will be exposed; the controller detects resistor changes of the photoresistor and sends demolition warning to server. Structure diagram of the system is shown in figure 1. 


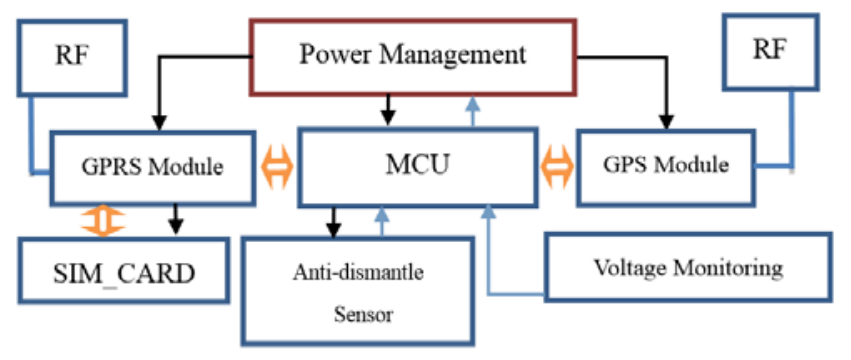

FIGURE I. STRUCTURE DIAGRAM OF THE SYSTEM

\section{SYSTEM HARDWARE DESIGN}

The design of the hardware system has six major parts, they are antenna unit, central control unit, GPS positioning module, GPRS communications module ${ }^{[4]}$, SIM card, system monitoring and power management.

\section{A. Central Control Unit}

MCU is responsible for the communication coordination among various modules of the system and the sensor data acquisition work, simple processing for all kinds of data, making the whole system safe and stable. STM8 series microcontroller into STM8A, STM8S, STM8L three series ${ }^{[5]}$, among these microcontroller, STM8L is a ultra-low power MCU. The current of STM8L151F3 microcontroller in low power mode is as low as $0.35 \mathrm{uA}$, as the main control chip of this system can greatly reduce the system static power consumption, ensuring long working hours of the system. MCU peripheral circuit is very simple and it only needs an external crystal vibration to work normally. The STM8L151F3 minimum system is shown in figure 2 .

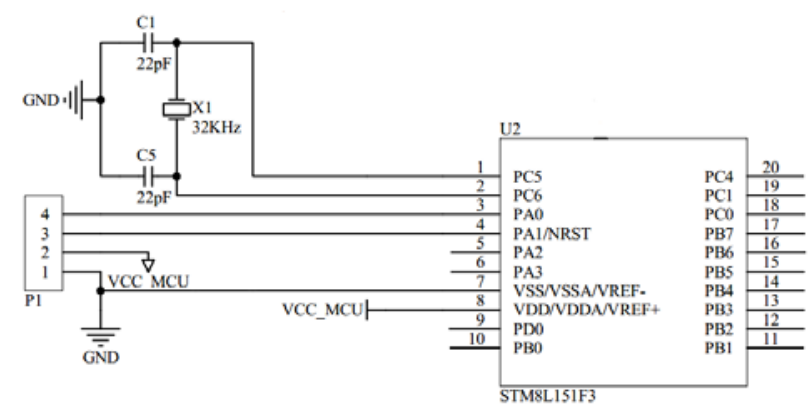

FIGURE II. STM8L151F3 MINIMUM SYSTEMS

\section{B. GPS Module}

GPS receiver module mainly completes the automobile real-time GPS data receiving and is one of the key parts of the receiver. It has many modules and different functions, and is generally mainly composed of low noise block down-converter, parallel signal channel, CPU, memory, etc ${ }^{[6]}$. As shown in figure 3, through its receiving antenna for satellite signals, with frequency conversion, amplification, smoothing and relating, mixing and a series of processing, the tracking, locking and measurement of satellite in the antenna horizon can be realized. After obtaining the position information of satellite and measuring the satellite signal propagation time, the antenna position can be calculated.
Tracking positioning terminal uses the L70 - R module, L70 - $\mathrm{R}$ is a small low-power GPS module, its power consumption is low to $13 \mathrm{~mA}$ in tracking mode and supports for multiple power saving modes, including Standby, Backup, Periodic modes and AlwaysLocate ${ }^{\mathrm{TM}}$ technology. In addition, it also supports LOCUS technology, which automatic recording log information into internal NVRAM. This module has high sensitivity(-165dBm@Tracking, 148dBm@Acquisition), with 66 capturing channels, 22 tracking and PRN 210 channels, supporting QZSS, DGPS, and SBAS, with strong anti-interference ability, its positioning accuracy is $2.5 \mathrm{~m}$ $\mathrm{CEP}^{[7]}$.

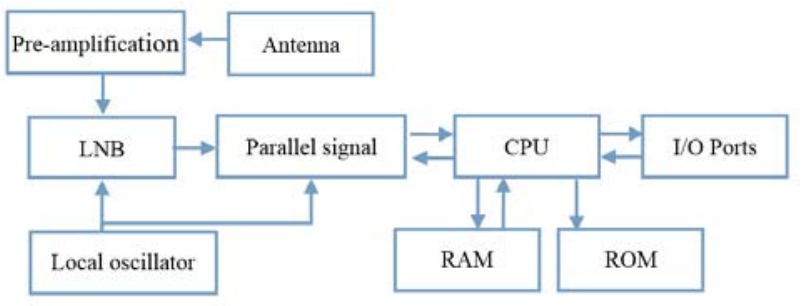

FIGURE III. GPS RECEIVING MODULE INTERNAL STRUCTURE

\section{GPRS Module}

The GPRS module will transmit the data receiving from the main control unit to the remote server system. This design adopts the domestic M26 module. The module supports 850, 900, 1800 and $1900 \mathrm{MHZ}$ band, its standby current is low to $1.3 \mathrm{~mA}$, embedding web service protocol stack (TCP/IP), supporting multiple Socket and IP address. M26 module supports GSM 07.07, 07.05 and the other AT extension instructions. The highest data transfer rate is that: 85.6 KPS (downward), 85.6 KPS (upward).

In addition, the tracking positioning terminal needs a SIM card as identity recognition of mobile communications and transport information encryption, and M26 module reserves special SIM card interface and also supply the power for SIM card, which greatly simplifies the design of the system.

\section{SYSTEM SOFTWARE DESIGN AND IMPLEMENTATION}

\section{A. Software System Structure}

The main functions of wireless GPS tracking terminal are that: for the achievement of reporting location information once a day and sending warning information and reminders to the remote server terminal in special cases. So tracking terminal needs to be completed of GPS information receiving, mobile wireless communications and monitoring abnormal events, finally realizing the stable and reliable operation of the system. Design of the system software program mainly includes the following several large structures:

The compiling of the software program are that: the implementation of initialization of the control system, controlling GPS and GPRS two modules to complete data collection and sending, the acquisition and processing for system abnormal signal, the end is that sending integrated information to the control center via mobile network system. System information processing program flow chart is shown in figure 4. 


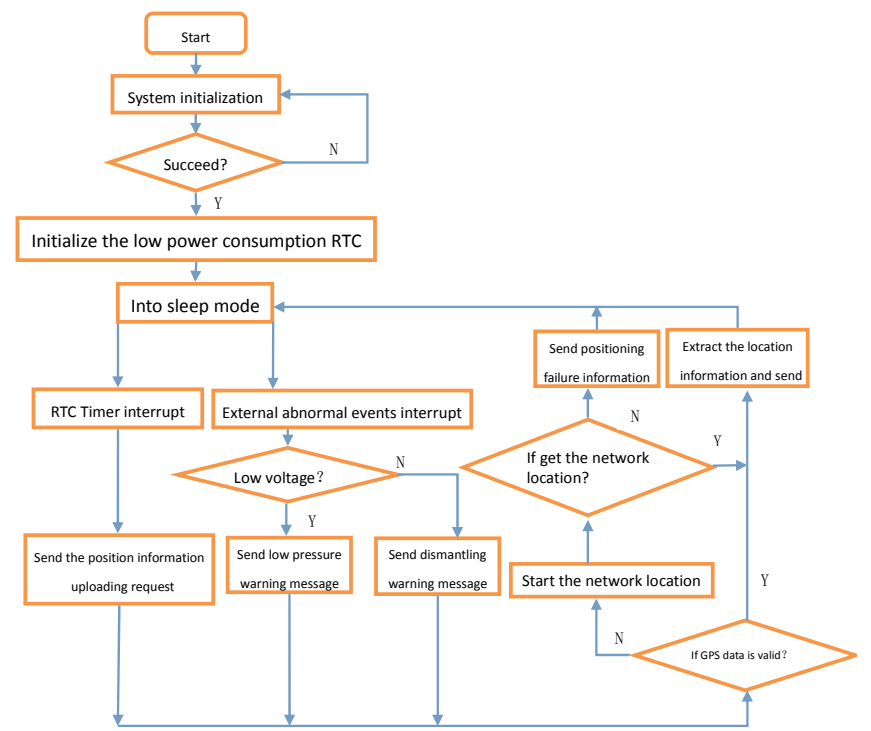

FIGURE IV.SYSTEM INFORMATION PROCESSING PROGRAM FLOW CHART

\section{B. System Software Design}

System initialization is mainly about completing the initialization of each MCU module: setting the internal RC oscillator as MCU system clock source, the system clock frequency is $16 \mathrm{MHz}$, the ADC conversion clock cycles is system 2 frequency division, a single conversion channel is 12, reference voltage is the internal reference voltage $1.224 \mathrm{~V}$, TIME4 clock is set as 128 frequency division of the system clock and the enable overflow interrupt is opened.

STM8L151F3 has low power RTC (Real - Time Clock) module, and can realize automatic timing wake function; There are two kinds of situations in this system will wake up the MCU from sleeping, one is that the RTC system will wake up MCU to read the GPS information and sent to the remote server every 24 hours, then it will come into sleeping state; another is abnormal events waking up, abnormal events includes two situations, namely that the MCU is under voltage and photosensitive resistance detection device is removed. The MCU will automatically report the location information and send warning information to the remote server in these cases.

\section{TCP/IP Flow of GSM Module}

The wireless communication of this system is carried out through GPRS mode, it involves the TCP/IP communication protocol, the basic software control flow chart is shown in figure 5.

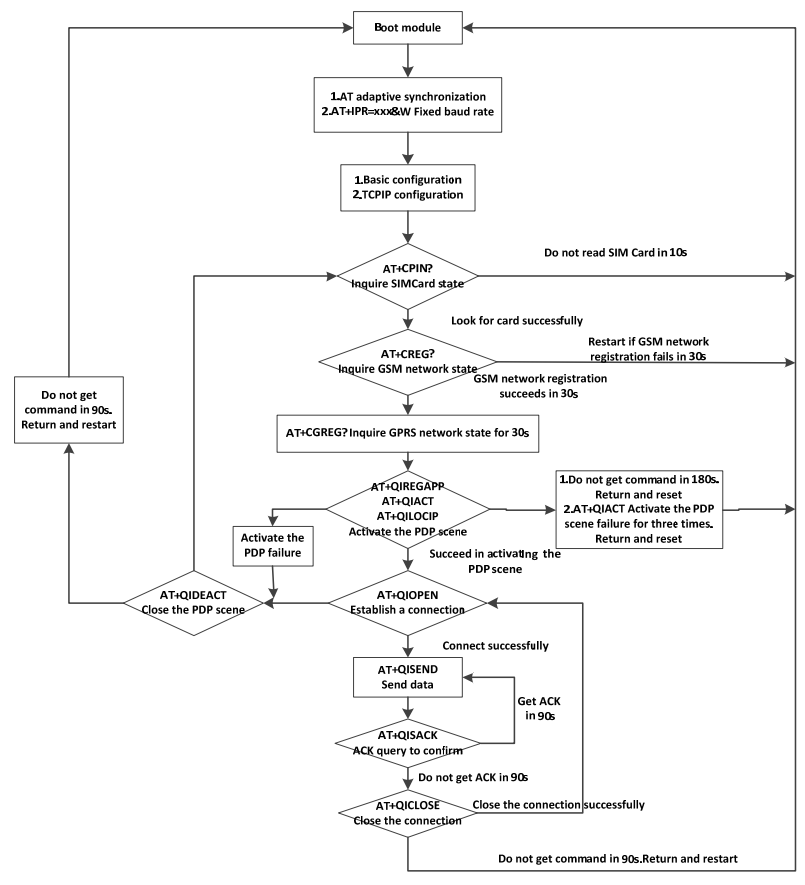

FIGURE V. PROCESS OF TCP/IP PROTOCOL

\section{CONCLUSION}

The paper introduces the main principle of the system application, and specially introduces working principle and design idea of the main module in low power spy type wireless GPS positioning system. This system has changed the mode which traditional module continues to send information, greatly reducing the overall power consumption of the system. Meanwhile, this paper carries on detailed analysis and optimization for various modules, strictly controlling the size of the entire terminal and unnecessary energy losses. Base station positioning cooperates with GPS positioning, improving the success rate and accuracy of positioning. Through the actual test the device achieves the requirement of design.

\section{REFERENCE}

[1] Jain K, Goel R. GPS Based Low Cost Intelligent Vehicle Tracking System (IVTS)[J]. International Proceedings of Computer Science \& Information Tech, 2012.

[2] Zhigang Liu. Intelligent GPS Vehicle Monitoring and Management System [D]. Shandong University, 2007.

[3] Minliang Yan, Yijun Miao. The Architecture and Implementation of Double Location Tracking Sysetem Basee on Base Station Positioning and GPS[J]. Electronic Products, 2012, (12):59-61.

[4] Wenmin Cai. Design and Implementation of Positioning System Based on A - GPS[D]. Xian University of Electronic Science and Technology,2010.

[5] Phe M P. Product information[J]. Eetimes Com, 2005, (7): 55-56.

[6] Tianying Chen. GPS/CDMA Location Tracking System of Automobile Monitoring Device[J]. Auto Electric Parts, 2011, (7):27-32.

[7] Shanghai Quectel communication co., LTD's website. http://www.quectel.com/cn/

[8] Lei Yang, Zhong-liang Deng, Lu Yin, Gang Sun. GNSS Fast Acquisition Algorithm Based on Maximum Likelihood Visibility Estimation. The Second China satellite Navigation Electronic Corpus Academic Conference.2011.

[9] Zhaoyuan Ouyang. Wireless Location Technology Research and Application Based on 3G[D]. South China University of Technology, 2011. 